\title{
A REAL VALUED HOMOMORPHISM ON ALGEBRAS OF DIFFERENTIABLE FUNCTIONS
}

\author{
JUAN ARIAS-DE-REYNA
}

(Communicated by R. Daniel Mauldin)

\begin{abstract}
In this paper we prove that, for every homomorphism $A$ on $C^{k}(E)$, there exists $x \in E$ such that $A(f)=f(x)$ for $f \in C^{k}(E)$. Here $C^{k}(E)(k=1,2, \ldots, \infty)$ denotes the algebra of all $k$-times differentiable real functions on a real and separable Banach space $E$.
\end{abstract}

Introduction. Let $C^{k}(E)(k=1,2, \ldots, \infty)$ be the algebra of all $k$-times differentiable function $f: E \rightarrow \mathbb{R}$ defined on a real Banach space $E$. Since $C^{k}(E)$ is an algebra of functions it makes sense to ask if, to each nonzero homomorphism $A: C^{k}(E) \rightarrow \mathbf{R}$, there corresponds $x \in E$ such that $A(f)=f(x)$ for all $f \in C^{k}(E)$, in other words, if $A$ is an evaluation.

If $k=0$, the answer is affirmative if and only if $E$ is realcompact. It turns out, since $E$ is a metric space, that it is realcompact if and only if $\operatorname{card}(E)$ is nonmeasurable (cf. Gillman and Jerison [1, pp. 226-232]).

On the other hand Jaramillo [4] defining two topologies $\tau_{\omega}^{k}$ and $\tau_{\delta}^{k}$ on $C^{k}(E)$, have proved that, if $\tau_{\omega}^{k}=\tau_{\delta}^{k}$, then every homomorphism on $C^{k}(E)$ is an evaluation. They have also proved that $\tau_{\omega}^{k}=\tau_{\delta}^{k}$ if $E$ has nonmeasurable cardinal and $E$ admits $B^{k}$ partitions of unity. To see what kind of Banach spaces admits $B^{k}$ partitions of unity, see Wells [6] and Sundaresan and Swaminatan [5].

In this paper we generalize the result of Jaramillo to all separable Banach spaces. We give a direct proof without using any topology on $C^{k}(E)$ nor partitions of unity on $E$.

1. Associated filter to a real homomorphism. In the following, $A$ : $C^{k}(E)$ $\rightarrow \mathbb{R}$ denotes a nonzero homomorphism. For $\alpha \in \mathbf{R}$, let $\hat{\alpha}$ be the constant function that associates the value $\alpha$ to each element $x \in E$. We claim that $A(\hat{\alpha})=\alpha$ for every $\alpha \in \mathbb{R}$. Indeed, $A(\hat{1}) \neq 0$ because $A \neq 0$. Then $B: \mathbb{R} \rightarrow \mathbf{R}$ defined by $B(\alpha)=A(\hat{\alpha})$ is a nonzero homomorphism from $\mathbf{R}$ into $\mathbf{R}$. Therefore $B$ is the identity homomorphism, that is $A(\hat{\alpha})=\alpha$ for every $\alpha \in \mathbf{R}$.

PROPOSITION 1. Let $A: C^{k}(E) \rightarrow \mathbb{R}$ be a nonzero homomorphism. For every $f \in C^{k}(E)$, the set $\{x \in E: A(f)=f(x)\}$ is nonempty.

Proof. Suppose $\{x \in E: A(f)=f(x)\}=\varnothing$. Then $f-\widehat{A(f)}$ is a real function, which does not take the value 0 . So $(f-\widehat{A(f)})^{-1} \in C^{k}(E)$ and we see that

$$
1=A(\hat{\mathbf{1}})=A\left((f-\widehat{A(f)})(f-\widehat{A(f)})^{-1}\right)=A(f-\widehat{A(f)}) A\left((f-\widehat{A(f)})^{-1}\right)
$$

which contradicts $A(f-\widehat{A(f)})=A(f)-A(f)=0$.

Received by the editors October 6, 1987.

1980 Mathematics Subject Classification (1985 Revision). Primary 46J15, 46J20; Secondary $41 \mathrm{~A} 65$. 
Proposition 2. Let $A: C^{k}(E) \rightarrow \mathbb{R}$ be a nonzero homomorphism. Denote by $S\left(f_{1}, \ldots, f_{n}\right)$ the set $\left\{x \in E: A\left(f_{i}\right)=f_{i}(x), i=1,2, \ldots, n\right\}$. The collection $\mathfrak{S}$ of all the sets $S\left(f_{1}, \ldots, f_{n}\right)$ where $f_{1}, \ldots, f_{n} \in C^{k}(E)$ is a filter basis on $E$.

PROOF. Since $S\left(f_{1}, \ldots, f_{n}\right) \cap S\left(g_{1}, \ldots, g_{m}\right)=S\left(f_{1}, \ldots, f_{n}, g_{1}, \ldots, g_{m}\right)$, it suffices to prove that $S\left(f_{1}, \ldots, f_{n}\right) \neq \varnothing$ for every $f_{1}, \ldots, f_{n} \in C^{k}(E)$.

Consider the function $\left.g=\sum_{i=1}^{n}\left(f_{i}-\widehat{A\left(f_{i}\right.}\right)\right)^{2} \in C^{k}(E)$. By Proposition 1, there exists $x \in E$ such that $A(g)=g(x)$. From the definition of $g$ we get $A(g)=0$, so that $\sum\left(f_{i}(x)-A\left(f_{i}\right)\right)^{2}=0$ which forces $f_{i}(x)=A\left(f_{i}\right)$ for every $i=1,2, \ldots, n$.

DEFINITION 3. Let $A: C^{k}(E) \rightarrow \mathbf{R}$ be a nonzero homomorphism. We will say that the filter $\mathscr{F}$ generated by the filter basis $\mathfrak{S}$, from Proposition 2, is the filter associated to $A$.

Proposition 4. Let $A: C^{k}\left(\mathbf{R}^{n}\right) \rightarrow \mathbf{R}$ be a nonzero homomorphism. Then there exists $x \in \mathbb{R}^{n}$ such that for every $f \in C^{k}\left(\mathbb{R}^{n}\right), A(f)=f(x)$.

PROOF. Let $f \in C^{k}\left(\mathbb{R}^{n}\right)$ be the function defined by $f\left(x_{1}, \ldots, x_{n}\right)=x_{1}^{2}+x_{2}^{2}+$ $\cdots+x_{n}^{2}$. The filter $\mathscr{F}$ associated to $A$ contains the set $K=\left\{\left(x_{1}, \ldots, x_{n}\right) \in\right.$ $\left.\mathbb{R}^{n}: A(f)=x_{1}^{2}+x_{2}^{2}+\cdots+x_{n}^{2}\right\}$. Since $K$ is compact, there exists $x \in \mathbb{R}^{n}$ adherent to $\mathscr{F}$.

For every $g \in C^{k}\left(\mathbf{R}^{n}\right)$ we see that $x \in \overline{\left\{y \in R^{n}: A(g)=g(y)\right\}}=\left\{y \in \mathbf{R}^{n}: A(g)=\right.$ $g(y)\}$. Hence $A(g)=g(x)$.

2. Main theorems. Now we construct some differentiable real functions on a Banach space, which we will use later.

LEMMA 5. Let $E$ be a real Banach space, $\left(x_{n}^{*}\right)$ a sequence in the dual space $E^{*}$ of $E$ which tends to zero in the weak ${ }^{*}$ topology, $\delta>0$ and $\varphi \in C^{\infty}(\mathbf{R})$ such that $\varphi(x)=0$ if $|x|<\delta$. Then we can define $\phi(x)=\sum_{n=1}^{\infty} \varphi\left(x_{n}^{*}(x)\right)$ and we have $\phi \in C^{\infty}(E)$.

PROOF. By hypothesis $x_{n}^{*}$ tends to zero in the weak* topology. It follows that $\lim x_{n}^{*}(x)=0$ for every $x \in E$. Moreover $\varphi(t)=0$ if $|t|<\delta$, so that $\sum_{n=1}^{\infty} \varphi\left(x_{n}^{*}(x)\right)$ is finite and we can define $\phi(x)$.

Now we will prove that, to each $a \in E$, there correspond a neighborhood $V$ of $a$ and a natural number $N$ such that, for every $x \in V, \phi(x)=\sum_{n=1}^{N} \varphi\left(x_{n}^{*}(x)\right)$. That is, in the neighborhood $V$ of $a$, we have $\phi=\psi \circ J$ where $J: E \rightarrow \mathbb{R}^{N}$ is the continuous linear map defined by $J(x)=\left(x_{1}^{*}(x), \ldots, x_{N}^{*}(x)\right)$ and $\psi: \mathbf{R}^{N} \rightarrow \mathbf{R}$ is defined in the form $\psi\left(t_{1}, \ldots, t_{N}\right)=\sum_{i=1}^{N} \varphi\left(t_{i}\right)$. In other words, we will prove that $\phi$ is a composition of infinitely differentiable functions. Therefore $\phi$ is infinitely differentiable too.

Suppose $a \in E$. We know that $\lim x_{n}^{*}(a)=0$. Then there exists $N$ such that $n>N$ implies $\left|x_{n}^{*}(a)\right|<\delta / 2$. Moreover, as $x_{n}^{*} \rightarrow 0$ in the weak ${ }^{*}$ topology, there exists $M$ such that $\left\|x_{n}^{*}\right\| \leq M$ for every $n$. Let $V$ be the ball of center $a$ and radius $\delta / 2 M$. If $x \in V,\|x-a\|<\delta / 2 M$. So for every $n>N$ we have $\left|x_{n}^{*}(x)\right| \leq \mid x_{n}^{*}(x-$ $a)|+| x_{n}^{*}(a) \mid<\delta / 2+\delta / 2=\delta$, and $\varphi\left(x_{n}^{*}(x)\right)=0$. Thus $\phi(x)=\sum_{n=1}^{N} \varphi\left(x_{n}^{*}(x)\right)$.

Proposition 6. Let $E$ be a real and separable Banach space and $A: C^{k}(E) \rightarrow$ $\mathbf{R}$ a nonzero homomorphism. There exists a $\in E$ such that, for every differentiable 
function $\varphi$ of the form $\psi \circ J$, where $\psi \in C^{\infty}\left(\mathbb{R}^{n}\right)$ and $J: E \rightarrow \mathbb{R}^{n}$ is a continuous linear map, we have $A(\varphi)=\varphi(a)$.

PROOF. Let $u: E^{*} \rightarrow \mathbb{R}$ be the linear form defined by $u\left(x^{*}\right)=A\left(x^{*}\right)$. First we will show that there exists $a \in E$ such that, for every $x^{*}, u\left(x^{*}\right)=x^{*}(a)$. It is the same to prove that $u$ is weak* continuous. Since $E$ is complete and separable, we apply a consequence of Grothendieck's completeness theorem (cf. Horvath [2, p. 253]) and it remains to prove that, if $x_{n}^{*} \rightarrow 0$ in the weak* topology, then $u\left(x_{n}^{*}\right) \rightarrow 0$.

First notice that, if $x_{1}^{*}, \ldots, x_{n}^{*} \in E^{*}$ and $\psi \in C^{\infty}\left(\mathbb{R}^{n}\right)$, then $A\left(\psi\left(x_{1}^{*}(x), \ldots, x_{n}^{*}(x)\right)\right.$ $=\psi\left(u\left(x_{1}^{*}\right), \ldots, u\left(x_{n}^{*}\right)\right)$. Indeed, let $J: E \rightarrow \mathbb{R}^{n}$ be the continuous linear map defined by $J(x)=\left(x_{1}^{*}(x), \ldots, x_{n}^{*}(x)\right)$. There exists an homomorphism of algebras $B: C^{\infty}\left(\mathbb{R}^{n}\right) \rightarrow C^{\infty}(E)$ that applies $\psi$ into $\psi \circ J$. The composition $A \circ$ $B: C^{\infty}\left(\mathbb{R}^{n}\right) \rightarrow \mathbb{R}$ is a nonzero homomorphism. Thus by Proposition 4 , there exists $\left(\alpha_{1}, \ldots, \alpha_{n}\right) \in \mathbb{R}^{n}$ such that $A \circ B(\psi)=\psi\left(\alpha_{1}, \ldots, \alpha_{n}\right)$, that is $A(\psi \circ J)=$ $\psi\left(\alpha_{1}, \ldots, \alpha_{n}\right)$ for every $\psi \in C^{\infty}\left(\mathbb{R}^{n}\right)$. Now if we consider the function $\psi_{i}$ defined by $\psi_{i}\left(t_{1}, \ldots, t_{n}\right)=t_{i}$ we obtain $A\left(x_{i}^{*}\right)=\alpha_{i}=u\left(x_{i}^{*}\right)$. Therefore

$$
A\left(\psi\left(x_{1}^{*}(x), \ldots, x_{n}^{*}(x)\right)=A(\psi \circ J)=\psi\left(u\left(x_{1}^{*}\right), \ldots, u\left(x_{n}^{*}\right)\right) .\right.
$$

Now let $\left(x_{n}^{*}\right)$ be a sequence in $E^{*}$ which tends to zero in the weak ${ }^{*}$ topology. We have to prove that $\lim u\left(x_{n}^{*}\right)=0$.

Fix $\delta>0$. Choose $\varphi \in C^{\infty}(\mathbb{R}), \varphi \geq 0, \varphi(t)=0$ if $|t|<\delta$ and $\varphi(t)=1$ if $|t|>2 \delta$. By Lemma 5, we can consider the function $\phi(x)=\sum_{n=1}^{\infty} \varphi\left(x_{n}^{*}(x)\right) \in$ $C^{\infty}(E)$. Now for every natural number $N$, we define the function $g_{N}$ in the form $g_{N}(x)=\phi(x)-\sum_{n=1}^{N} \varphi\left(x_{n}^{*}(x)\right)$. Since $g_{N}(x) \geq 0$ Proposition 1 can be applied. It shows that there exists $\bar{x}$ such that $A\left(g_{N}\right)=g_{N}(\bar{x}) \geq 0$. Now (1) leads to

$$
A(\phi) \geq A\left(\sum_{n=1}^{N} \varphi\left(x_{n}^{*}(x)\right)\right)=\sum_{n=1}^{N} \varphi\left(u\left(x_{n}^{*}\right)\right) \text {. }
$$

This result being valid for every $N$ and observing that $\varphi\left(u\left(x_{n}^{*}\right)\right) \geq 0$, we conclude that the series $\sum_{n=1}^{\infty} \varphi\left(u\left(x_{n}^{*}\right)\right)$ converges absolutely. Hence we have $\left|u\left(x_{n}^{*}\right)\right|<2 \delta$ except for a finite number of indices. Thus the linear form $u$ is weak ${ }^{*}$ continuous, so that there exists $a \in E$ such that $u\left(x^{*}\right)=x^{*}(a)$ for every $x^{*} \in E^{*}$.

Finally if $\varphi=\psi \circ J$ we obtain, by (1),

$$
A(\varphi)=\psi\left(u\left(x_{1}^{*}\right), \ldots, u\left(x_{n}^{*}\right)\right)=\psi\left(x_{1}^{*}(a), \ldots, x_{n}^{*}(a)\right)=\varphi(a)
$$

and the proof is complete.

Proposition 7. Let $E$ be a real Banach space, $\left(x_{n}^{*}\right)$ a sequence in the dual space $E^{*}$ and $\varphi \in C^{\infty}(\mathbb{R})$ such that $\varphi(t)=1$ if $|t|>1$. Then there exists a sequence $\left(\alpha_{n}\right)$ of positive real numbers so that $\phi(x)=\sum_{n=1}^{\infty} \alpha_{n} \varphi\left(x_{n}^{*}(x)\right)$ defines a function $\phi \in C^{\infty}(E)$.

ProOF. Fix $x^{*} \in E^{*}$ and denote by $\left(x^{*}\right)^{n}$ the multilinear map defined by $\left(x^{*}\right)^{n}\left(x_{1}, \ldots, x_{n}\right)=x^{*}\left(x_{1}\right) x^{*}\left(x_{2}\right) \cdots x^{*}\left(x_{n}\right)$ in $\mathscr{L}(E, E, \ldots, E ; \mathbf{R})$. We see that the $n$th derivative of $\varphi \circ x^{*}$ is given by $D^{n}\left(\varphi \circ x^{*}\right)(x)=D^{n} \varphi\left(x^{*}(x)\right)\left(x^{*}\right)^{n}$.

Since $\varphi(t)=1$ if $|t|>1$ we obtain that there exist constants $M_{n}$ such that $\left|D^{n} \varphi\left(x^{*}(x)\right)\right| \leq M_{n}$. Hence

$$
\left\|D^{n}\left(\varphi \circ x^{*}\right)(x)\right\|_{\mathscr{L}(E, \ldots, E ; \mathbf{R})} \leq M_{n}\left\|x^{*}\right\|^{n} .
$$


In order to prove that the series $\sum \alpha_{n} \varphi\left(x_{n}^{*}(x)\right)$ converges, it suffices to choose the sequence $\left(\alpha_{n}\right)$ such that $\sum\left|\alpha_{n}\right| M_{0}<+\infty$. Moreover the function $\phi$ is differentiable if we choose the sequence $\left(\alpha_{n}\right)$ such that series $\sum\left|\alpha_{n}\right| M_{1}\left\|x_{n}^{*}\right\|$ converges, and $\phi$ is of class $C^{\infty}$ if for every natural number $p$ the series $\sum\left|\alpha_{n}\right| M_{p}\left\|x_{n}^{*}\right\|^{p}$ converges. But it is obvious that we can choose the sequence $\left(\alpha_{n}\right)$ verifying these conditions, which completes the proof.

THEOREM 8. Let $E$ be a real and separable Banach space and $A: C^{k}(E) \rightarrow \mathbb{R}$ a nonzero homomorphism. Then there exists a point $a \in E$ such that $A(\varphi)=\varphi(a)$ for every $\varphi \in C^{k}(E)$.

ProOF. We know, by Proposition 6, that there exists $a \in E$ such that, if $\varphi \in C^{\infty}(E)$ is of the form $\varphi=\psi \circ J$, where $\psi \in C^{\infty}\left(\mathbb{R}^{n}\right)$ and $J: E \rightarrow \mathbb{R}^{n}$ is a continuous linear map, then $A(\varphi)=\varphi(a)$.

Since $E$ is separable, there exists a sequence $\left(x_{n}^{*}\right)$ in the dual space such that $\|x\|=\sup \left|x_{n}^{*}(x)\right|$ for every $x \in E$. To prove this, let $\left(a_{n}\right)$ be a dense sequence in $E$ and choose $x_{n}^{*}$ in the dual space such that $\left\|x_{n}^{*}\right\|=1$ and $x_{n}^{*}\left(a_{n}\right)=\left\|a_{n}\right\|$. Now $\left|x_{n}^{*}(x)\right| \leq\|x\|$ for every $n$ and, on the other hand, to each $\varepsilon>0$, there corresponds $a_{n}$ such that $\left\|x-a_{n}\right\|<\varepsilon$. Thus we have $\|x\| \leq\left\|a_{n}\right\|+\left\|x-a_{n}\right\| \leq \varepsilon+x_{n}^{*}\left(a_{n}\right) \leq$ $2 \varepsilon+\left|x_{n}^{*}(x)\right|$.

Now let $f \in C^{k}(E)$. As $f$ is continuous in $a$, given $\varepsilon>0$, there exists $\delta>0$ such that $\|x-a\| \leq \delta$ implies $|f(x)-f(a)|<\varepsilon$.

Next we choose a function $\varphi \in C^{\infty}(\mathbb{R})$ such that $\varphi(t) \neq 0$ if $|t|>\delta, \varphi(t)=0$ if $|t| \leq \delta, \varphi(t)=1$ if $|t|>1$, and $\varphi(t) \geq 0$ for every $t \in \mathbb{R}$. Now Proposition 7 can be applied. It shows that there exists a sequence $\left(\alpha_{n}\right)$ of positive real numbers such that the function $\psi(x)=\sum_{n=1}^{\infty} \alpha_{n} \varphi\left(x_{n}^{*}(x-a)\right) \in C^{\infty}(E)$. In the same way the function $\phi(x)=\sum_{n=1}^{\infty}\left(\alpha_{n} / n\right) \varphi\left(x_{n}^{*}(x-a)\right) \in C^{\infty}(E)$.

Observe that, for $\|x-a\| \leq \delta, \phi(x)=0$ since $\left|x_{n}^{*}(x-a)\right|<\delta$. Moreover, if $\|x-a\|>\delta$, there exists a natural number $n$ such that $\left|x_{n}^{*}(x-a)\right|>\delta$, so that $\phi(x)>0$.

Our next objective is to prove that $A(\phi)=0$. Fix $N$. By Proposition 2, there exists $x \in E$ such that $A(\phi)=\phi(x), A\left(x_{i}^{*}\right)=x_{i}^{*}(a)=x_{i}^{*}(x)$ for every $i=1,2, \ldots, N$ and $A(\psi)=\psi(x)$. It follows that

$$
A(\psi)=\psi(x)=\sum_{n=1}^{\infty} \alpha_{n} \varphi\left(x_{n}^{*}(x-a)\right)=\sum_{N+1}^{\infty} \alpha_{n} \varphi\left(x_{n}^{*}(x-a)\right) \geq 0
$$

and

$$
A(\phi)=\phi(x)=\sum_{n=1}^{\infty}\left(\alpha_{n} / n\right) \varphi\left(x_{n}^{*}(x-a)\right)=\sum_{N+1}^{\infty}\left(\alpha_{n} / n\right) \varphi\left(x_{n}^{*}(x-a)\right) .
$$

Therefore $0 \leq N A(\phi) \leq A(\psi)$. As this is valid for every $N$, we conclude that $A(\phi)=0$.

Finally, by Proposition 2, there exists $y \in E$ such that $A(f)=f(y)$ and $0=$ $A(\phi)=\phi(y)$. Since $\phi(y)=0$, we have $\|y-a\|<\delta$. It turns out, by the choice of $\delta$, that $|f(y)-f(a)|<\varepsilon$. Hence what we have proved is that, for every $\varepsilon>0$, the inequality $|A(f)-f(a)|<\varepsilon$ holds. This completes the proof. 


\section{BIBLIOGRAPHY}

1. L. Gillman and M. Jerison, Rings of continuous functions, Van Nostrand, Princeton, N. J., 1960.

2. J. Horvath, Topological vector spaces, Addison-Wesley, Reading, Mass., 1966.

3. T. Husain, Multiplicative functionals on topological algebras, Pitman, Boston, Mass., 1983.

4. J. A. Jaramillo Algebras de funciones continuas y differenciables. Homomorphismos $e$ interpoblación, Tesis Doctoral, Universidad Complutense de Madrid, 1987.

5. K. Sundaresan and S. Swaminatan, Geometry and nonlinear analysis in Banach spaces, Lecture Notes in Math., vol. 1131, Springer, 1985.

6. J. C. Wells, Differentiable functions on Banach spaces with Lipschitz derivatives, J. Differential Geometry 8 (1973), 135-152.

Analisis Matematico, Universidad de Sevilla, Sevilla, Spain

Current address: Facultad de Matemáticas, Apdo. 1160, 41080-Sevilla, Spain 